\title{
Standard spirometry to assess emphysema in patients with chronic obstructive pulmonary disease: the Emphysema Severity Index (ESI)
}

\author{
Roberto W. Dal Negro, ${ }^{1}$ Matteo Paoletti, ${ }^{2}$ Massimo Pistolesi ${ }^{2}$ \\ ${ }^{I}$ National Centre for Respiratory Pharmacoeconomics and Pharmacoepidemiology - CESFAR, Verona \\ ${ }^{2}$ Department of Experimental and Clinical Medicine, University of Florence, Italy
}

\begin{abstract}
Background: Chronic obstructive pulmonary disease (COPD) is a generic term identifying a condition characterized by variable changes in peripheral airways and lung parenchyma. Standard spirometry cannot discriminate the relative role of conductive airways inflammatory changes from destructive parenchymal emphysema changes. The aim of this study was to quantify the emphysema component in COPD by a simple parameter (the Emphysema Severity Index ESI), previously proved to reflect CT-assessed emphysema.

Methods: ESI was obtained by fitting the descending limb of MEFV curves by a fully automated procedure providing a 0 to 10 score of emphysema severity. ESI was computed in COPD patients enrolled in the CLIMA Study.

Results: The vast majority of ESI values ranged from 0 to 4, compatible with no-to-mild/moderate emphysema component. A limited proportion of patients showed ESI values $>4$, compatible with severe-to-very severe emphysema. ESI values were greatly dispersed within each GOLD class indicating that GOLD classification cannot discriminate emphysema and conductive airways changes in patients with similar airflow limitation. ESI and diffusing capacity $\left(\mathrm{DL}_{\mathrm{CO}}\right)$ were significantly correlated $(\mathrm{p}<0.001)$. However, the great dispersion in their correlation suggests that ESI and $\mathrm{DL}_{\mathrm{CO}}$ reflect partially different anatomo-functional determinants in COPD.

Conclusions: Airflow limitation has heterogenous determinants in COPD. Inflammatory and destructive changes may combine in CT densitometric alterations that cannot be detected by standard spirometry. ESI computation from spirometric data helps to define the prevailing pathogenetic mechanism underlying the measured airflow limitation. ESI could be a reliable advancement to select large samples of patients in clinical or epidemiological trials, and to compare different pharmacological treatments.
\end{abstract}

Key words: COPD; airflow limitation; Emphysema Severity Index; ESI; phenotypes.

Correspondence: Roberto W. Dal Negro, National Center for Respiratory Pharmacoeconomics and Pharmacoepidemiology, via G. Rossetti 4, 37124 Verona, Italy. E-mail: robertodalnegro@gmail.com

Contributions: Authors equally contributed to the study. RWD, MPi planned the study and wrote the manuscript; MPa carried out the ESI computation and statistical calculations. All the authors have read and approved the final version of the manuscript and agreed to be accountable for all aspects of the work.

Conflict of interest: The authors declare no conflict of interest in the present investigation. RWD is Associate Editor of Multidisciplinary Respiratory Medicine; MPi is a member of the Editorial Board of Multidisciplinary Respiratory Medicine.

Ethics approval: The project and the study design were approved by the Ethical and Scientific Commission of Research and Clinical Government in the session of June $16^{\text {th }}, 2020$ (cod. \# ESI-1/2020).

Consent for publication: Not applicable.

Availability of data and materials: The data used to support the findings of this study are available from the corresponding author upon reasonable request. 


\section{Introduction}

Emphysema is anatomically characterized by variable changes in peripheral airways and destruction of lung parenchyma. Beside the airflow limitation shared with other clinical conditions globally unified under the term of chronic obstructive pulmonary disease (COPD), emphysema is functionally characterized by greater increase of static lung volumes and reduction of $\mathrm{CO}$ diffusing capacity. When compared to patients with predominant involvement of conductive airways (such as, chronic bronchitis, small airway disease), patients with COPD and predominant emphysema component are usually characterized by a different clinical profile (i.e., lower BMI, more pronounced dyspnea, lower prevalence of cough and sputum, and lower tendency to develop right heart decompensation) [1-5]. In particular, patients claiming dyspnea as their prevailing symptom showed a 3.4 times higher probability to belong to the emphysema phenotype rather than to the simple obstructive phenotype where lung function is more preserved in the great majority of cases [5]. Moreover, the annual drop of lung function is much greater in emphysema [6,7] that was also described as a powerful independent predictor of respiratory and nonrespiratory mortality $[8,9]$. At present, standard spirometry is the most used method for diagnosing COPD and for checking patients to recruit in clinical, epidemiological and pharmacologic studies. However, spirometry can only measure the overall degree of airway obstruction and cannot discriminate the relative role of disorders in conductive airways from those typical of parenchymal destruction due to emphysema [10-12]. Consequently, as these two pathogenetic determinants are frequently coexisting in COPD patients, their relative role remains undefined by spirometry in the great majority of cases.

Chest computed tomography (CT) has been recently suggested as a reliable and suitable alternative methodology for detecting and quantifying in vivo the extent of parenchymal emphysematous destruction in COPD, as well as the bronchial wall thickening [1315]. Dedicated software analysis by the co-registration of inspiratory and expiratory CT, and the recent introduction of artificial neural networks allowed to define whether the emphysematous destruction rather than the conductive airways disease is the predominant pathogenetic mechanism in COPD [16-20]. At present, CT scan is also regarded as an excellent imaging biomarker for implementing personalized medicine in COPD [21]. However, the complexity and the reduced availability of these methods for wide clinical use, together to their high cost and their induced-radiation load, limited the extensive feasibility of CT scan for investigating COPD, that is one of the most prevalent chronic disorders in the general population.

A recent study showed that mathematical modeling of the descending limb of the maximal expiratory flow volume (MEFV) curve provides the Emphysema Severity Index (ESI). It approximated very well the scores obtained by means of CT in COPD and proved suitable enough for both clinical and research application [22]. The ability of ESI to identify the emphysema component and to assess its severity has been further validated by comparison with the CT-quantified emphysema in a very large multiethnic population of smokers of the COPDgene study [23].

The aim of the present study was to assess the extent of emphysema by ESI computation in COPD patients recruited for the CLIMA (CLInical MAnifestation of COPD) study, that is a large, national, multicenter trial that was specifically designed for the first time in Italy with the aim of assessing the prevalence of the most frequent COPD phenotypes and defining their clinical and lung function profiles [5].

\section{Patients and Methods}

The patients' sample consisted of the 1,012 COPD patients recruited in the CLIMA study [males $77.0 \%$; mean age $=71.5$ year \pm 8.8 SD (min-max: 41-92 years); mean BMI $=27.42 \pm 5.63$ (minmax: 23.6-31.0; never smokers were 9.1\%, while active smokers $21.2 \%$ ] [5]. The multicenter study involved twenty-four pneumological sites evenly distributed throughout Italy, and the sample had been previously calculated in order to be representative of the whole Italian COPD population. COPD severity by GOLD class in the sample was: class $1=13.4 \%$; class $2=41.2 \%$; class $3=32.9 \%$, and class $4=12.5 \%[5]$.

Further to anagraphics, patients' history, clinical signs, and some critical biological data (blood eosinophil count; total blood $\mathrm{IgE}$, and $\mathrm{alpha}_{1}$ anti-trypsin levels), several lung function variables (static and forced volumes and flows; \% short-term $\mathrm{FEV}_{1}$ reversibility from baseline 30' after salbutamol $400 \mathrm{mcg}$; DLco \% predicted; $\mathrm{SpO}_{2}$, and BODE Index) were collected. Moreover, four specific questionnaires (the Modified Questionnaire of British Medical Research Council - mMRC; the COPD Assessment Test - CAT; the Borg Category Scale - BCS, and the Quality-of-Life Questionnaire - EQ5D-5L) were also administered in order to check the patients' physical performance, quality of life, and impact. Finally, the description of the chest X-ray was also recorded, paying particular attention to the clear mention of "emphysema" in the radiological report [5].

\section{ESI methodology}

The ESI computation is based on a biomechanical model approximating the shape of the descending limb of the maximum expiratory flow/volume (MEFV) curve. The computation assumes that at a given time the pressure drop of a fluid flowing in a cylindrical duct resembling the airways is directly related to the specific friction factor, to the density and the velocity of the fluid, and inversely related to the diameter of the duct. Details of the theoretical and biomechanical background are reported in previous publications [22,23].

The mathematical computation of ESI is obtained by a specific software which was designed to fit the descending limb of the MEFV curve by a fully automated procedure, thus providing realtime results suitable for clinical practice and for large data-sets in clinical and pharmacologic trials. Being directly related to the shape of the curve of each patient, ESI does not require standardization for the input variables, i.e., \% predicted calculations. The on-line computation of ESI is available from the URL https:/www.emphysema.app/. The app using discrete input variables as absolute values (PEF, $\mathrm{FEF}_{25}, \mathrm{FEF}_{50}, \mathrm{FEF}_{75}, \mathrm{FVC}$ ) obtained by standard spirometry provides a numerical output ranging from 0 to 10 that classifies each patient according to the presence and severity of emphysema, as validated by the comparison with CT scan quantitative data. Descriptive statistics are presented as means and standard deviation. The relationship between ESI and lung function parameters was assessed by Pearson's $r$ correlation. A $\mathrm{p}<0.05$ was assumed as significant.

\section{Results}

The ESI methodology was applied in 903/1,012 patients of the CLIMA study in whom all the five numeric spirometric parameters needed to calculate the ESI score were available. Patients' age, sex, anthropometric data, and lung function data are reported in Table 1. The distribution of the ESI values within the 903 patients 
investigated is reported in Figure 1. ESI values are distributed in the whole range of variation, thus indicating that emphysema may be identified at variable extent in this COPD population. The vast majority of ESI values ranged from 0 to 4 , compatible with no-tomild/moderate emphysema component. A limited proportion of patients had ESI values higher than 4, compatible with severe-tovery severe emphysema component of their flow limitation.

Figure 2 reports the GOLD classification [24] of the 809 patients in whom both $\mathrm{FEV}_{1} \%$ and $\mathrm{FEV}_{1} / \mathrm{FVC}$ were available. As occurring in the majority of COPD patients included in large clinical and pharmacologic trials, GOLD 2 and 3 were the most prevalent classes of severity also in the present study, approximatively entailing two third of the whole patients' sample. These data confirm those of the CLIMA study obtained by a multiparametric lung function approach. In the CLIMA study, the prevalence of the emphysema COPD phenotype was $38.8 \%$, and a wide distribution among different phenotypes was anyway observed [5].

The great dispersion of ESI within each of the four GOLD classes is clearly shown in Figure 3, in which means and percentiles of ESI are considered, and in Figure 4, in which the individual ESI values are reported for each patient. The distribution of ESI values in these two figures demonstrates that GOLD classification does not allow to discriminate among patients characterized by similar levels of expiratory airflow limitation, and to discriminate those without significant emphysema from those in whom emphysema of various severity degrees is concomitant. Moreover, it also appears that GOLD 2 patients may have ESI values up to 4-5, thus reflecting the occurrence of moderate emphysema at CT scan. The whole range of emphysema severity (from absent to very severe) as assessed by $\mathrm{CT}$ is mirrored by the ESI values measured in GOLD stage 3 and 4 patients.

Figure 5 clearly further confirms that the GOLD classification does only reflect the global level of expiratory airflow limitation

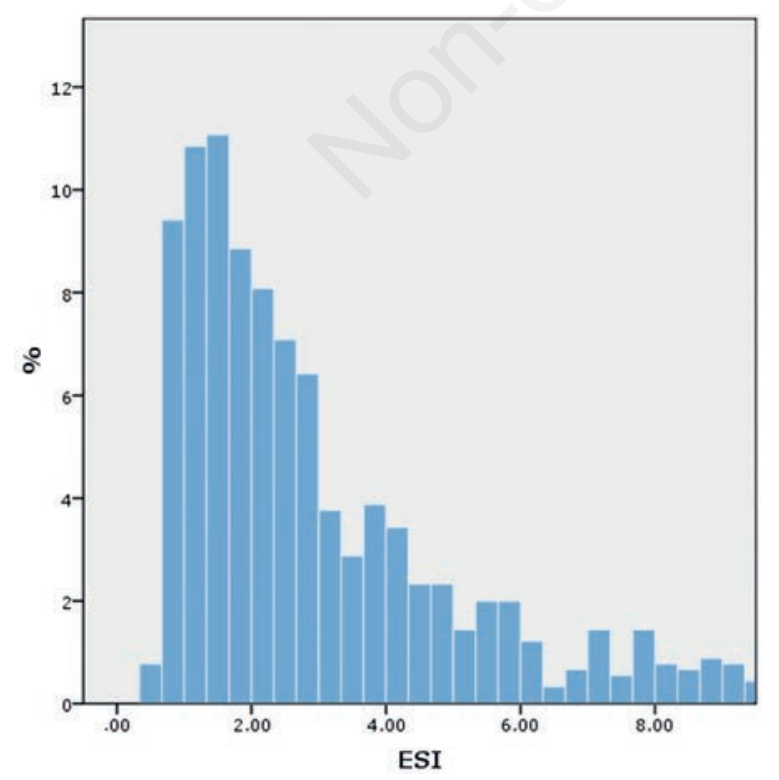

Figure 1. Percent distribution of patients according to ESI values. that may be due either to changes in conductive airways or emphysema parenchymal destruction. It is also evident that patients' classification according to the GOLD classes shows a lower dispersion of data when compared with the level of obstruction as reflected by $\mathrm{FEV}_{1} / \mathrm{FVC}$.

It clearly appears that the easy implementation of ESI computation to the standard spirometry allows a much better approximation of the relative contribution of the involvement of conductive airways and/or the emphysema component to the overall airflow limitation, as merely assessed by routine spirometry.

The correlation between ESI values and values of $\mathrm{CO}$ diffusing capacity (\% predicted) was calculated in 542/1,012 patients in whom both measurements were available, and corresponding results have been reported in Figure 6. Although the correlation proved significant $(\mathrm{r}=0.29, \mathrm{p}<0.001)$, individual data were widely scattered,

Table 1. Age, sex, BMI, lung function data and ESI values in the whole sample.

\begin{tabular}{lccc} 
Variable & $\mathbf{n}$ & Mean & SD \\
Age (males/females) & 903 & 69.4 & 9.2 \\
& $614 / 289$ & & \\
$\mathrm{BMI}$ & & 26.9 & 5.1 \\
\hline $\mathrm{FEV}_{1} \%$ & 56 & 23 \\
$\mathrm{FEV}_{1} / \mathrm{FVC}$ & 0.53 & 0.14 \\
$\mathrm{DL}_{\mathrm{CO}} \%$ & & 65.3 & 27.3 \\
$\mathrm{ESI}$ & & 3.2 & 2.5 \\
\hline
\end{tabular}

BMI, body mass index; $\mathrm{FEV}_{1} \%$, \% predicted forced expiratory volume in the first second; FVC, forced vital capacity; $\mathrm{DL}_{\mathrm{C} 0} \%$, \% predicted diffusing capacity for carbon monoxide; ESI, emphysema severity index.

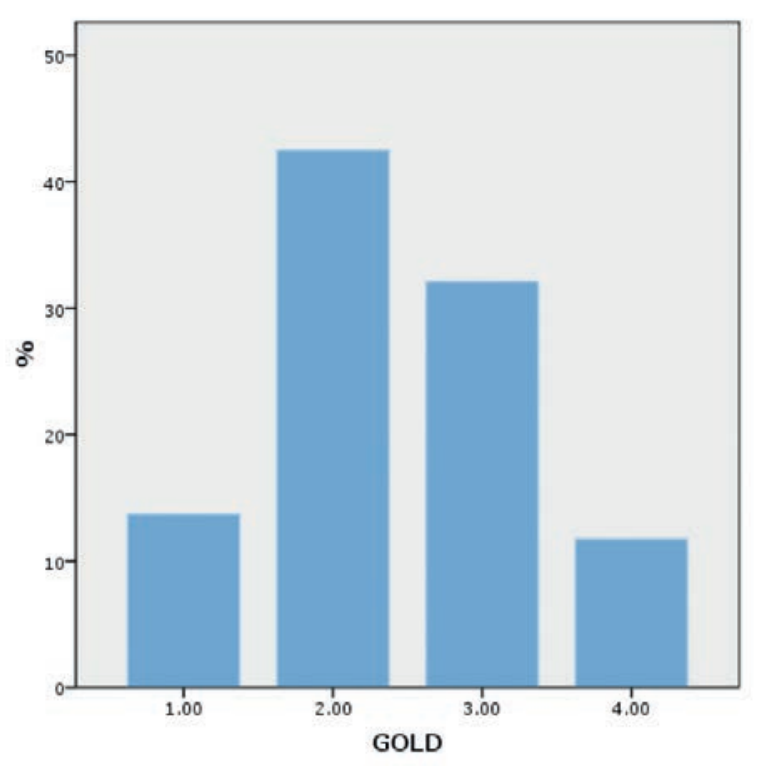

Figure 2. Percent distribution of patients according to the GOLD classification. 
thus suggesting that ESI and diffusing capacity partially rely to different anatomical and functional alterations in COPD. This scattering rate is significantly $(\mathrm{r}=0.36)$ reduced only when patients with a $\mathrm{FEV}_{1}$ value $<1$ liter $(\mathrm{n}=190)$ are excluded from calculations. In general. the dispersion of ESI values increases progressively in proportion to the reduction of diffusing capacity.

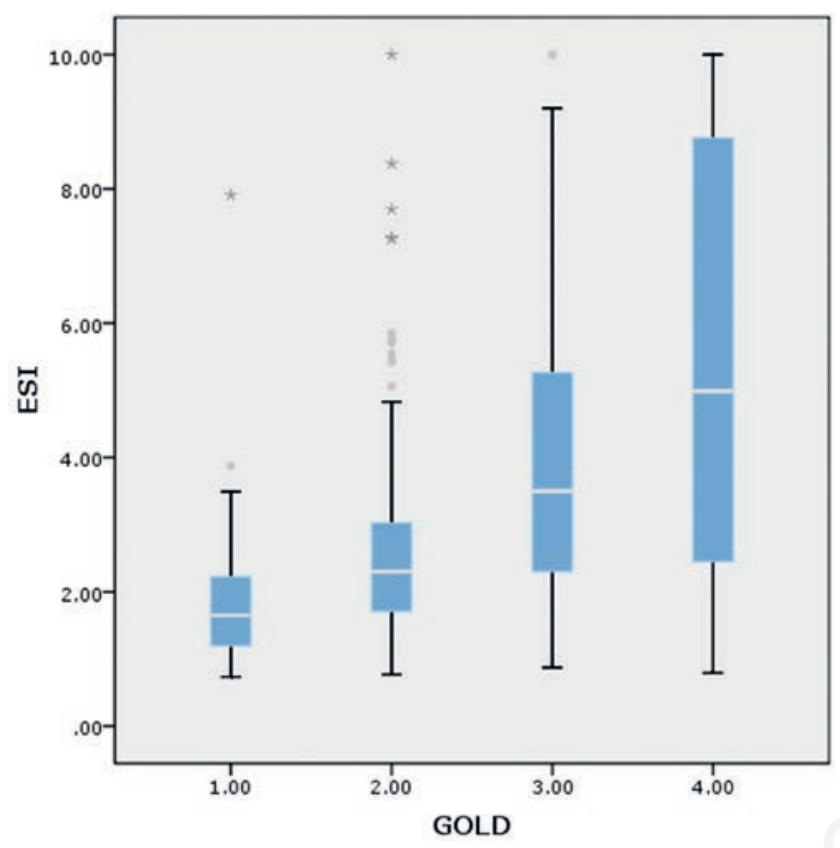

Figure 3. Distribution of means and percentiles of ESI values according to GOLD classification.

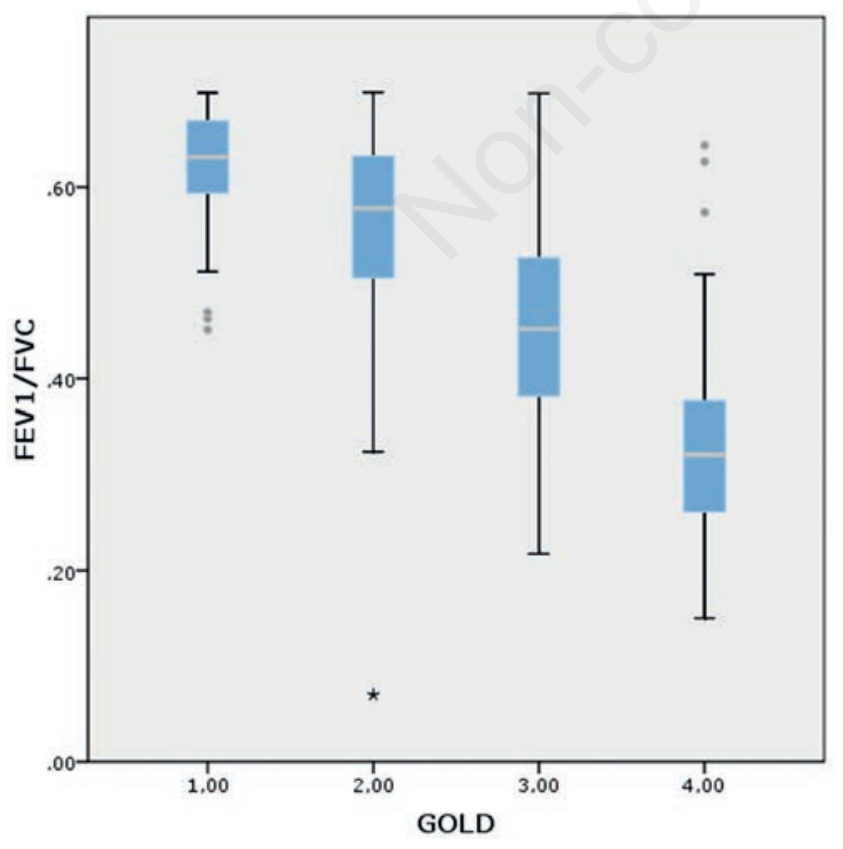

Figure 5. Distribution of $\mathrm{FEV}_{1} / \mathrm{FVC}$ means and percentiles according to GOLD classes (out-liners are reported).

\section{Discussion}

There is a consolidate evidence that what is currently defined "COPD" corresponds to various respiratory conditions that can be variably characterized in clinical, biological, and lung function terms, the so-called COPD phenotypes. Despite the well-known heterogeneity in clinical presentations of COPD, the exact identi-

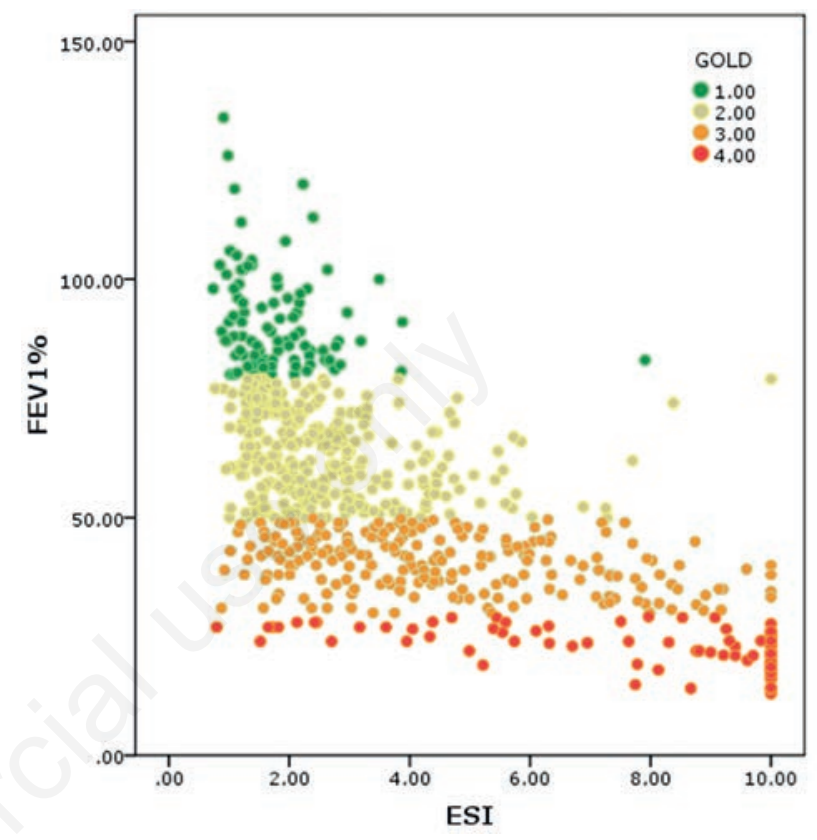

Figure 4. Relationship between individual values of $\mathrm{FEV}_{1} \%$ and ESI.

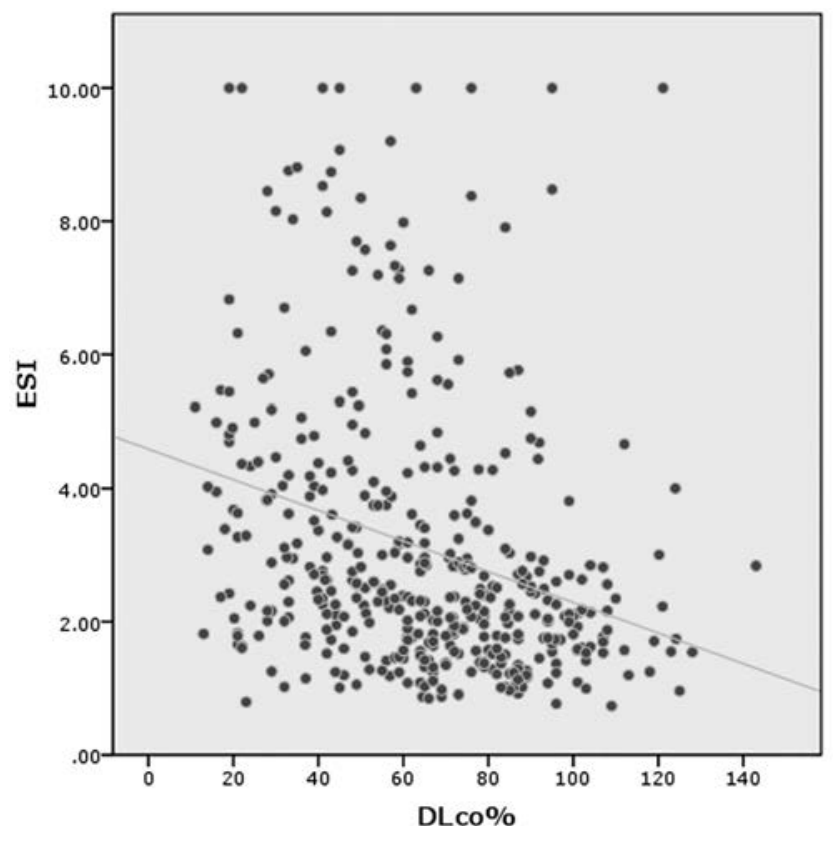

Figure 6. Relationship between ESI and DLco (\% pred.) values. 
fication of the pathogenetic mechanisms underlying the current airflow limitation, in many cases variably mixed, is not usually pursued in real world clinical practice [25]. Moreover, a further crucial issue is the identification of the peculiar lung function profiles corresponding to these manifestations.

Although the existing consensus on the great impact of the phenotypical approach to COPD, the message currently emerging from the literature is that the spirometric staging alone (the most widely used tool) is not sensitive enough to describe the complexity of COPD and to characterize the lung function pattern of the corresponding phenotypes [5,10-12]. Unfortunately, measurements of static lung volumes and of $\mathrm{CO}$ diffusing capacity (that would permit a much more objective and appropriate evaluation of emphysema contribution to airflow obstruction) are largely underused both in clinical practice and in clinical trials due to their complexity, higher cost, and unavailability. Actually, when the relative contribution of inflammatory changes in conductive airways and of the parenchimal destructive component is known for each COPD patient, the interpretation whether the pathologic mechanism underlying the expiratory obstruction is due to increased airflow resistance or to reduced elastic recoil is possible and much easier. Without CT scan use, this information can be achieved, as it has been shown in the CLIMA study by means of a multiparametrical lung function approach [5].

Stemming from this evidence, the opportunity of assessing and discriminating the different pathogenetic components in COPD patients (such as the contribution of airway obstruction rather than of parenchymal destruction) by means of a simple, low cost, and widely available lung function test (i.e., the standard spirometry) would represent a paramount opportunity in both clinical practice and for large clinical, epidemiological and pharmacological studies (when CT is usually not available), with the final aim to personalize the most appropriate pharmacologic respiratory treatment. The chance to apply the ESI computation to the large sample of patients of the CLIMA Study represented a good opportunity for better investigating and understanding the pathophysiologic mechanisms underlying the airflow limitation in COPD, and also represented a pivotal investigational model for prospective and/or retrospective large scale studies where COPD patients are merely recruited by standard spirometry.

The present study clearly shows that the GOLD classification, according to its definition, is only related to the level of obstruction, but does not give information as to whether emphysema is present or not, and, if present, at what extent. ESI measurements prove that the extent of parenchymal destructive changes, that are typical of emphysema, are greater in GOLD 3 and 4 patients. In these cases, their multiparametrical lung function profile, physical performance, and quality of life are much poorer when compared to those of patients belonging to the simple COPD phenotype [5]. However, the scattering of the ESI correlation data tends to emphasize that significant levels of emphysema may even occur in GOLD 1 and 2 COPD patients, but also that a quite limited parenchymal destruction may also occur in a very small proportion of GOLD 3 and 4 COPD patients. The disperse correlation between ESI and DLco is confirmed by a previous observation showing that diffusing capacity could be excessively low in patients with $\mathrm{FEV}_{1}$ values $<1$ liter with no or minimal emphysema on CT [26].

Just in order to proceed beyond $\mathrm{FEV}_{1}$, even if the measure of DLco confirms probative indeed in favor of the presence of the emphysema component in COPD phenotype, the reduction in DLco is related to the dysfunction of the lung alveolar-capillary surface that could be not only a consequence of its destruction (as in the case emphysema and reflected by ESI), but also of the occur- rence of the uneven distribution of ventilation within normal functioning alveoli, as caused by significant inflammatory and obstructive changes in the extreme peripheral airways $[5,27,28]$.

Data of the present study are a further confirmation that airway flow limitation has a multifactorial etiology in COPD. This evidence can explain why heterogeneous clinical, functional and therapeutic response are frequently obtained in patients with the same or very similar levels of airway obstruction, as assessed by spirometry. In a paper aimed to investigate the relationship existing between $\mathrm{CT}$ and lung function parameters it was indeed shown that densitometric data and lung function variables are not linearly related over the whole range of COPD severity [29]. In this study, CT densitometric changes were related to the reduction in diffusing capacity and to the increase in hyperinflation measurements only in more severe COPD patients, and by airflow obstruction measurements only in the less severely affected patients [29].

In our opinion, the opportunity to provide such kind of information on the COPD endotype by means of the simple ESI computation that is quickly obtained from standard spirometry should be regarded as an important step forward to improve the understanding of the structural changes underlying COPD. It should also be emphasized that ESI is only based on the MEFV curve morphology and, consequently, it is independent from \% predicted values of lung function parameters measured in the MEFV curves. The knowledge that the severity of emphysema as assessed by CT may vary widely within the same GOLD stage is a notion widely held [30]. This information may be of great relevance in clinical practice when needing to personalize the therapeutic strategy of COPD, and standard spirometry is the only operational tool available.

From this point of view, it was shown that, in general, COPD patients are the most pharmacologically treated and that a great proportion of them do not receive the appropriate, personalized respiratory treatment also in Italy [31]. This evidence tends to further emphasize the still too poor assessment of the lung function profile of different COPD phenotypes, particularly that one characterized by the emphysema component. Unfortunately, the current treatment of COPD [24] still is not based on the assessment of the pathophysiologic changes underlying expiratory airflow limitation, nor on their site of lesions, but rather just on the severity of symptoms claimed and the frequency of exacerbations. These variables are difficult to assess objectively and they are not related to the underlying mechanism of flow limitation [6].

The still great debate as to whether COPD patients do benefit or not from the addition of inhaled steroids to bronchodilators may possibly be minimized by assessing the role of the parenchymal destructive component. ESI computation might play a crucial role from this point of view as it permits to define whether, and at what extent, the parenchymal destruction due to emphysema contributes to the expiratory airflow limitation, spirometrically measured. In fact, patients with high values of ESI probably cannot obtain maximal benefits from steroid treatment since mainly affected from emphysema, while steroids should be likely more effectively prescribed in patients with increased resistance of the conductive airways due to inflammatory changes.

The evidence that the destruction of lung parenchyma is not homogeneously distributed in COPD patients characterized by equal or similar severity of their original airway flow limitation might contribute to explain why some big pharmacological trials proved somewhat limited and provided conflicting results in terms of outcomes. Actually, the different prevalence of emphysema in these COPD populations can make the difference. Inclusion or exclusion of COPD patients with a significant emphysema component could have a great influence on the final trials' results 
designed and calibrated for specific outcomes. For instance, the lack of a significant increase in survival observed in the TORCH study [32] after treatment with inhaled salmeterol and fluticason might be due to excess mortality secondary to the unrequired steroid effect causing pneumonia in patients with significant emphysema, not investigated at all in that study [33,34].

For these reasons, the retrospective post hoc ESI computation in COPD patients of previous big trials might consent to revisit and critically redefine the outcomes. In other words, at what degree of emphysema severity the therapeutic strategy based on the double bronchodilation would have been more appropriate than that one based on adding inhaled steroids?

The present study has some limitations. First, ESI computation was calculated retrospectively in a large study sample in which ESI had not been originally included in the variables to collect. Second, as in all large trials, CT densitometry was not available in the CLIMA study, and we assumed that ESI represents parenchymal destruction without reference standard measurements. However, the accuracy of ESI in mirroring accurately the occurrence of emphysema damage by comparison with CT densitometry had already been previously validated in a very large population of smokers and COPD patients [23].

The present study also has some points of strength. First, ESI computations were carried out on a large sample of COPD patients accurately investigated with the aim to define the prevalence of different COPD phenotypes (the CLIMA Study). Second, the patients' sample was representative of the whole Italian COPD population. Third, ESI values were in substantial concordance with data from the CLIMA Study that was carried out by assessing a complete lung function, and not merely by standard spirometry. Fourth, ESI measurements are particularly suitable and convenient for large trials in which accuracy is required, but times and costs represent substantial limiting factors of the investigational methods.

\section{Conclusions}

Data of the present study further support the evidence that COPD is a very heterogeneous disorder in which inflammatory and destructive changes may variably combine [28]. These phenomena can lead to densitometric alterations measurable by CT [35] that standard spirometric assessment of airway limitation is not able to clarify in terms of underlying causes.

The ESI computation, such as the availability of a quick parameter easily obtained by an algorithm developed to fit the descending limb of the flow/volume curve by PEF, $\mathrm{FEF}_{25}, \mathrm{FEF}_{50}$, $\mathrm{FEF}_{75}$, and FVC expressed in absolute value, can be implemented in clinical, pharmacologic and epidemiologic trials to assess the contribution of the emphysema component in each COPD patient to recruit.

The implementation of ESI measurements may reveal whether and at what extent inflammatory changes of the conductive airways rather than the parenchymal destruction are the prevailing pathogenetic mechanism, even if the two mechanisms may variably coexist in a significant proportion of COPD patients. However, the knowledge of their relative contribution easily achievable by standard spirometry usually employed to detect the level of obstruction is of paramount importance, both for therapeutic decisions and for the prognostic assessment of COPD.

The availability of ESI, that is an objective quantitative index independent of the \% predicted MEFVs values, easily derived from standard spirometry, and significantly correlated with the CT quantitative assessment of emphysema, should be regarded as a reliable advancement either in clinical terms for quick definition of the emphysema component in COPD patients, or for selecting large samples of patients (i.e., clinical or epidemiological trials) in short time and at low cost in order to check or compare their pharmacological treatments when standard spirometry is the only possible lung function test to perform.

\section{Acknowledgments}

The authors are very grateful to A. Menarini I.F.R. for the unrestricted grant in support of the study.

\section{References}

1. Burrows B, Fletcher CM, Heard BE, Jones NL, Wootliff JS he emphysematous and bronchial types of chronic airways obstruction. A cliniopathological study of patients in London and Chicago. Lancet 1966;87:830-35.

2. Pistolesi M, Camiciottoli G, Paoletti M, Marmai C, Lavorini F, Meoni E, et al. Identification of a predominant COPD phenotype of COPD in clinical practice. Respir Med 2008;102:36776.

3. Camiciottoli G, Bigazzi F, Paoletti M, Cestelli L, Lavorini F, Pistolesi M. Pulmonary function and sputum characteristics predict computed tomography phenotype and severity of COPD. Eur Respir J 2013;42:626-35.

4. Ogawa E, Nakano Y, Ohara T, Muro S, Hirai T, Sato S, et al. Body mass index in male patients with COPD: correlation with low attenuation areas on CT. Thorax 2009;64:20-5.

5. Dal Negro RW, Carone M, Cuttitta G, Gallelli L, Pistolesi M, Privitera S, et al. Prevalence and clinical features of most frequent phenotypes in the Italian COPD population: the CLIMA Study. Multidiscip Respir Medi 2021;16:790.

6. Nishimura M, Makita H, Nagai K, Konno S, Nasuhara Y, Hasegawa $\mathrm{M}$, et al. Annual change in pulmonary function and clinical phenotype in chronic obstructive pulmonary disease. Am J Respir Crit Care Med 2012;185:44-52.

7. Kurashima K, Takaku Y, Hoshi T, Kanauchi T, Nakamoto K, Takayanagi $\mathrm{N}$, et al. Lobe-based computed tomography assessment of airway diameter, airway or vessel number, and emphysema extent in relation to the clinical outcomes of COPD. Int J Chron Obstruct Pulmon Dis 2015;10:1027-33.

8. Haruna A, Muro S, Nakano Y, Ohara T, Hoshino Y, Ogawa E, et al. CT scan findings of emphysema predict mortality in COPD. Chest 2010;138:635-40.

9. Johannessen A, Skorge TD, Bottai M, Grydeland TB, Nilsen RM, Coxson H, et al. Mortality by level of emphysema and airway wall thickness. Am J Respir Crit Care Med 2013;187:6028.

10. Han MK, Agusti A, Calverly PM, Celli BR, Criner G, Curtis JL, et al. Chronic obstructive pulmonary disease phenotypes: the future of COPD. Am J Respir Crit Care Med 2010;182:598-604.

11. Macklem PT, Mead J. Resistance of central and peripheral airways measured by a retrograde catheter. J Appl Physiol 1967;22:395-401.

12. Lange P, Halpin DM, O'Connell DE, MacNee W. Diagnosis, assessment, and phenotyping of COPD beyond FEV1. Int J COPD 2016;11:3-12.

13. Madani A, De Maertelaer V, Zanen J, Gevenois PA. Pulmonary emphysema: radiation dose and section thickness at multidetector CT quantification--comparison with macroscopic and 
microscopic morphometry. Radiology 2007;243:250-7.

14. Hackx M, Bankier AA, Gevenois PA. Chronic obstructive pulmonary disease: CT quantification of airways disease. Radiology 2012;265:34-48.

15. Lynch DA, Austin JH, Hogg JC, Grenier PA, Kauczor HU, Bankier AA, et al. CT-definable subtypes of chronic obstructive pulmonary disease: A statement of the Fleischner Society. Radiology 2015; 277:192-205.

16. Galbán CJ, Han MK, Boes JL, Chughtai KA, Meyer CR, Johnson TD, et al. Computed tomography-based biomarker provides unique signature for diagnosis of COPD phenotypes and disease progression. Nat Med 2012;18:1711-5.

17. Bartholmai BJ, Raghunath S, Karwoski RA, Moua T, Rajagopalan S, Maldonado F, et al. Quantitative computed tomography imaging of interstitial lung diseases. J Thorac Imaging 2013; 28:298-307.

18. Raghunath S, Rajagopalan S, Karwoski RA, Maldonado F, Peikert T, Moua T, et al. Quantitative stratification of diffuse parenchymal lung diseases. PLoS One 2014;9:e93229.

19. Pompe E, van Rikxoort EM, Schmidt M, Rühaak J, Estrella LG, Vliegenthart R, et al. Parametric response mapping adds value to current computed tomography biomarkers in diagnosing chronic obstructive pulmonary disease. Am J Respir Crit Care Med 2015;191:1084-6.

20. Aerts HJ. The potential of radiomic-based phenotyping in precision medicine: A review. JAMA Oncol 2016;2:1636-42.

21. Frix AN, Cousin F, Refaee T, Bottari F, Vaidyanathan A, Desir $\mathrm{C}$, et al. Radiomics in lung diseases imaging: State-of-the-art for clinicians. J Pers Med 2021;11:602.

22. Occhipinti M, Paoletti M, Bartholmai BJ, Rajagopalan S, Karwoski RA, Nardi C, et al. Spirometric assessment of emphysema presence and severity as measured by quantitative $\mathrm{CT}$ and CT-based radiomics in COPD. Respir Res 2019;20:101.

23. Occhipinti M, Paoletti M, Crapo JD, Make BJ, Lynch DA, Brusasco V, et al. Validation of a method to assess emphysema severity by spirometry in the COPDGene study. Respir Res. 2020;21:103.

24. Singh D, Agusti A, Anzueto A, Barnes PJ, Bourbeau J, Celli $\mathrm{BR}$, et al. Global strategy for the diagnosis, management, and prevention of chronic obstructive lung disease: the GOLD science committee report 2019. Eur Respir J
2019; 18;53:1900164.

25. Hogg JC, Chu F, Utokaparch S, Woods R, Elliott WM, Buzatu $\mathrm{L}$, et al. The nature of small-airway obstruction in chronic obstructive pulmonary disease. N Engl J Med 2004;350:264553.

26. Gelb AF, Schein M, Kuei J, Tashkin DP, Müller NL, Hogg JC, et al. Limited contribution of emphysema in advanced chronic obstructive pulmonary disease. Am Rev Respir Dis 1993; 147:1157-61.

27. Vazquez JH, Garcia IA, Meca AA, de Andres AL, Ruix CM, Garcia MJB, de Miguel Diez J. COPD phenotypes: differences in survival. Int J COPD 2018;13:2245-51.

28. Karimi R, Tornling G, Forsslund H, Mikko M, Wheelock A, Nyrén $\mathrm{S}$, et al. Lung density on high resolution computer tomography (HRCT) reflects degree of inflammation in smokers.Respir Res 2014;15:23.

29. Paoletti M, Cestelli L, Bigazzi F, Camiciottoli G, Pistolesi M. Chronic obstructive pulmonary disease: Pulmonary function and CT lung attenuation do not show linear correlation. Radiology 2015;276:571-8.

30. Makita H, Nasuhara $Y$, Nagai K, Ito $Y$, Hasegawa M, Betsuyaku T, et al. Characterisation of phenotypes based on severity of emphysema in chronic obstructive pulmonary disease. Thorax 2007;62:932-7.

31. Dal Negro RW, Bonadiman L, Turco P, Tognella S, Iannazzo S. Costs of illness analysis in Italian patients with chronic obstructive pulmonary disease (COPD): an update. Clinicoecon Outcomes Res 2015;7:153-9.

32. Calverley PM, Anderson JA, Celli B, Ferguson GT, Jenkins C, Jones PW, et al; TORCH investigators. Salmeterol and fluticasone propionate and survival in chronic obstructive pulmonary disease. N Engl J Med 2007;356:775-89.

33. Chalmers JD, Keir HR. 10 years since TORCH: shining a new light on the risks of inhaled corticosteroids in COPD. Eur Respir J 2017;50:1701582.

34. Suissa S. Inhaled corticosteroids and pneumonia mortality in COPD patients. Eur Respir J 2019;54:1901276.

35. Occhipinti M, Paoletti M, Bigazzi F, Camiciottoli G, Inchingolo R, Larici AR, et al. Emphysematous and nonemphysematous gas trapping in chronic obstructive pulmonary disease: Quantitative CT findings and pulmonary function. Radiology 2018;287:683-92.

Received for publication: 10 September 2021. Accepted for publication: 26 October 2021.

This work is licensed under a Creative Commons Attribution-NonCommercial 4.0 International License (CC BY-NC 4.0).

(C) Copyright: the Author(s), 2021

Licensee PAGEPress, Italy

Multidisciplinary Respiratory Medicine 2021; 16:805

doi:10.4081/mrm.2021.805 